\title{
ANNALES ACADEMIAE SCIENTIARUM FENNICAE
}

Series A

I. MATHEMATICA

403

\section{SOME REMARKS ON PICARD SETS}

BY

KIKUJI MATSUMOTO

HEL SINKI 1967

S U O M A L A INEN TIEDEAKATEMIA

doi:10.5186/aasfm.1967.403 
Communicated 14 Octob er 1966 by OlLi Lehto and K. I. Virtanen 


\section{Some remarks on Picard sets}

1. By the definition in Lehto's paper [4], a Picard set $E$ means a totally disconnected closed set in the extended $z$-plane, in whose complementary domain $\Omega$ each single-valued meromorphic function $f(z)$ with at least one essential singularity in $E$ takes every value infinitely often except for at most two values. Our definition given in [7] is slightly different from Lehto's. We restricted the class of functions $f(z)$ in the above to that of functions $f(z)$ with $E$ as the set of essential singularities, that is, we called $E$ an $n$-Picard set, simply a Picard set for $n=2$, if each $f(z)$ with $E$ as the set of essential singularities has at most $n$ exceptional values at any singularity $\zeta \in E$. Now we call $E$ an $n$-Picard set in Lehto's sense if each $f(z)$ with at least one essential singularity in $E$ omits at most $n$ values in the intersection of $\Omega$ and every neighbourhood of any essential singularity $\zeta \in E$. We remark that omitted values may be taken by $f(z)$ at points of $E$ where $f(z)$ is meromorphic. Of course $n$-Picard set in Lehto's sense are ones in our sense, but, for instance, countable $E$ are Picard sets in our sense because $f(z)$ has isolated singularities, while they are not always Picard sets or even $n$-Picard sets $(n \geqq 3)$ in Lehto's sense. Indeed let $w_{1}, w_{2}, \ldots, w_{n}$ be finite and distinct and let $E$ be the union of all the $w_{i}$-points $(i=1,2, \ldots, n)$ of a non-rational entire function and the point at infinity. Then $E$ is not an $n$-Picard set in Lehto's sense.

As for the existence, Lehto [4] showed that all sufficiently thin countable sets with one limit point are Picard sets in his sense. For non-countable $E$, Carleson [2] gave examples of 3-Picard sets in Lehto's sense by means of Cantor sets. We [5], [6] also gave some sufficient conditions for $E$ to be $n$-Picard sets in Lehto's sense for $n \geqq 3$ and showed the existence of perfect sets satisfying the conditions. Further we showed that there exist perfect $n$-Picard sets in Lehto's sense $(n \geqq 3)$ which are not $(n-1)$ Picard sets. Recently we have succeeded to give Cantor sets which are Picard sets in our sense. We have proved that any Cantor set with successive ratios $\xi_{n}$ satisfying the condition

$$
\xi_{n+1}=\mathrm{o}\left(\xi_{n}^{2}\right)
$$

is a Picard set (see [7] and also [8]). But from our proof, we cannot see whether these Cantor sets are Picard sets in Lehto's sense or not. In this 
paper we shall show that Cantor sets are certainly Picard sets in Lehto's sense under a much stronger condition. We shall also show that Lehto's condition for countable set with one limit point to be Picard sets in his sense can be relaxed considerably.

2. First we shall be concerned with the countable case. Let $\left\{a_{n}\right\}_{n=1,2}, \ldots$ be a point set whose points converge to infinity and let $E$ denote the union of $\left\{a_{n}\right\}$ and the point at infinity. The result to be established is as follows:

Theorem 1. If the points of $\left\{a_{n}\right\}_{n=1,2, \ldots}$ satisfy the condition

$$
\left|a_{n}\right|^{3}=0\left(\left|a_{n+1}\right|\right),
$$

then $E$ is a Picard set in Lehto's sense.

Remark. Lehto [4] proved the same assertion under the stronger condition

$$
\left(\log \left|a_{n}\right|\right)^{2+\delta}=0\left(\log \left|a_{n+1}\right|\right) \quad(\delta>0) .
$$

3. Before proving the theorem, we give some lemmas. We shall consider the Riemann sphere $\Sigma$ with radius $1 / 2$ touching the $w$-plane at the origin. For $w$ and $w^{\prime}$ in the $w$-plane we denote by $\left[w, w^{\prime}\right]$ the chordal distance between them, that is,

$$
\left[w, w^{\prime}\right]= \begin{cases}\frac{\left|w-w^{\prime}\right|}{\sqrt{\left(1+|w|^{2}\right)\left(1+\left|w^{\prime}\right|^{2}\right)}} & \text { if } w \neq \infty \text { and } w^{\prime} \neq \infty \\ \frac{1}{\sqrt{1+|w|^{2}}} & \text { if } w^{\prime}=\infty .\end{cases}
$$

Further we denote by $C(w ; \delta)(\delta>0)$ the spherical open disc with center $w$ and with chordal radius $\delta$.

Let $w=f(z)$ be a single-valued regular function in an annulus $1<|z|<e^{\mu}(\mu>0)$ omitting two values 0 and 1 . Our first lemma is a consequence of Bohr-Landau's theorem [1]:

If $g(z)$ is regular in $|z|<1$ and $g(z) \neq 0,1$ there, then

$$
\max _{|z|=r}|g(z)| \leqq \exp \left(\frac{K \log (|g(0)|+2)}{1-r}\right) \text { for any } r, 0 \leqq r<1,
$$

where $K$ is a positive constant (a precise form of Schottky's theorem).

Lemma 1. There exists a positive constant $A$ such that the length, consequently the diameter of the image curve of $|z|=e^{\mu / 2}$ by $f(z)$ with respect to the chordal distance is dominated by $A e^{-\mu / 2}$ for sufficiently large $\mu$ (Carleson [2]. See also Matsumoto [6]). 
For completeness, we shall give the proof.

Proof. From Bohr-Landau's theorem, we can see easily that if $w=g(z)$ is regular in $1<|z|<e^{\sigma}(\sigma>0)$ satisfying the conditions

$$
g(z) \neq 0,1 \text { and } \min _{|z|=e^{\sigma / 2}}|g(z)|<a \text { for a positive } a,
$$

then there is a positive constant $b$ depending only on $a$ and $\sigma$ such that

$$
\max _{|z|=e^{\sigma / 2}}|g(z)| \leqq b .
$$

For a fixed $\sigma>0$, first we shall show, supposing $\mu>\sigma$, that there exists a positive number $\delta$ not depending on $\mu$ and $f(z)$ such that the images of $|z|=e^{\sigma / 2}$ and $|z|==e^{u-\sigma / 2}$ by $f(z)$ lie outside at least one of three dises $C(0 ; \delta), C(1 ; \delta)$ and $C(\infty ; \delta)$. In fact, let $z_{1}$ and $z_{2}$ be points on $|z|=e^{\sigma / 2}$ and $|z|=e^{u-\sigma / 2}$ respectively. Then $f\left(z_{1}\right)$ and $f\left(z_{2}\right)$ lie outside at least one of three dises $C\left(0 ; \delta^{\prime}\right), C\left(1 ; \delta^{\prime}\right)$ and $C\left(\infty ; \delta^{\prime}\right)$, where $\delta^{\prime}$ is a positive constant such that these three discs are mutually disjoint and hence can be taken independently of $\mu$ and $f(z)$. Suppose that $f\left(z_{1}\right)$ and $f\left(z_{2}\right)$ lie outside $C\left(\infty ; \delta^{\prime}\right)$, then by the fact mentioned above, we see that there is a positive $\delta_{\infty}$ such that the images of $|z|=e^{\sigma / 2}$ and $|z|=e^{u-\sigma / 2}$ lie outside $C\left(\infty ; \delta_{\infty}\right)$. Next suppose that $f\left(z_{1}\right)$ and $f\left(z_{2}\right)$ lie outside $C\left(1 ; \delta^{\prime}\right)$. Then we see using the linear transformation which transforms points $w=0, w=1$ and the point at infinity to points $w=1$, the point at infinity and $w=0$ respectively that there is a positive $\delta_{1}$ such that the images lie outside $C\left(1 ; \delta_{1}\right)$. Similarly we can find a positive $\delta_{0}$ and set

$$
\delta=\min \left\{\delta_{0}, \delta_{1}, \delta_{\infty}\right\}>0 .
$$

Obviously this $\delta$ satisfies our conditions.

Now by the maximum principle we see that the image of $e^{\sigma / 2} \leqq|z| \leqq e^{\mu-\sigma / 2}$ by $f(z)$ lies outside at least one of $C(0 ; \delta), C(1 ; \delta)$ and $C(\infty ; \delta)$. Since the chordal distance remains invariant under the linear transformation $\left(1+\bar{w}_{0} w\right) /\left(w-w_{0}\right)$ for any $u_{0} \neq \infty$, we may assume that this image lies outside $C(\infty ; \delta)$. Then

$$
|f(z)| \leqq M=\frac{\sqrt{1-\delta^{2}}}{\delta} \text { on } e^{\sigma / 2} \leqq|z| \leqq e^{n-\sigma / 2} .
$$

By Cauchy's integral theorem, we have

$$
f^{\prime}(z)=\frac{1}{2 \pi i}\left\{\int_{|\zeta|=e^{\mu-\sigma / 2}} \frac{f(\zeta)}{(\zeta-z)^{2}} d \zeta-\int_{|\zeta|=e^{\sigma / 2}} \frac{f(\zeta)}{(\zeta-z)^{2}} d \zeta\right\}
$$

for every $z$ on $|z|=e^{\mu / 2}$ and hence, if $\mu \geqq 2+\sigma$, 


$$
\left|f^{\prime}(z)\right| \leqq \frac{2 M}{\left(e^{(\mu-\sigma) / 2}-1\right)^{2}} \leqq \frac{2 e^{2+\sigma}}{(e-1)^{2}} M e^{-\mu} .
$$

Therefore we have

$$
\int_{|z|=e}^{\mu / 2}\left|f^{\prime}(z)\right||d z| \leqq \frac{2 e^{2+\sigma}}{(e-1)^{2}} M e^{-\mu} \cdot 2 \pi e^{\mu / 2}=\frac{4 \pi e^{2+\sigma}}{(e-1)^{2}} M e^{-\mu / 2} .
$$

Since the length of the image curve $f\left(|z|=e^{u / 2}\right)$ with respect to the metric $|d w|$ is not smaller than that with respect to the chordal distance, we can take $\left(4 \pi e^{2+\sigma} /(e-1)^{2}\right) M$ as $A$, for $M$ depends only on $\delta$ and $\sigma$ and hence does not depend on the function $f(z)$. Our lemma is proved.

4. Let $\Delta$ be a triply connected domain with boundary components $\Gamma_{1}, \Gamma_{2}$ and $\Gamma_{3}$ and let $f(z)$ be single-valued, regular and omitting two values 0 and 1 in $\Delta$. We assume that the images of $\Gamma_{1}, \Gamma_{2}$ and $\Gamma_{3}$ by $f(z)$ are contained in spherical dises $C_{1}, C_{2}$ and $C_{3}$ respectively and prove

Lemma 2. Let $\delta>0$ be so small that the three spherical discs $C(0 ; 2 \delta)$, $C(1 ; 2 \delta)$ and $C(\infty ; 2 \delta)$ are mutually disjoint. If the radii of $C_{1}, C_{2}$ and $C_{3}$ are less than $\delta / 2$, then either (1) $C_{1}, C_{2}$ and $C_{3}$ contain the origin, the point $w=1$ and the point at infinity one by one, so that they are contained in $C(0 ; \delta), C(1 ; \delta)$ and $C(\infty ; \delta)$ one by one and $f(z)$ takes each value outside the union of $C(0 ; \delta), C(1 ; \delta)$ and $C(\infty ; \delta)$ once and only once in $\Delta$, or (2) any one of them cannot be disjoint from the union of the other two, so that, by the maximum principle, there is a disc with radius less than $3 \delta / 2$ which contains the image of $\Delta$.

Proof. First we shall prove that $f(z)$ takes each value outside the union of $C(0 ; \delta), C(1 ; \delta)$ and $C(\infty ; \delta)$ once and orly once in 4 , if $C_{1}, C_{2}$ and $C_{3}$ are contained in these three discs one by one. We may assume without any loss of generality that $C_{1}, C_{2}$ and $C_{3}$ are contained in $C(0 ; \delta)$, $C(1 ; \delta)$ and $C(\infty ; \delta)$ respectively. Contrary suppose that $f(z)$ takes a value $w_{0}$ outside the union at two points $z^{\prime}$ and $z^{\prime \prime}$ in $\Delta$ and join $w_{0}$ to $C(0 ; \delta)$ and $C(\infty ; \delta)$ with two curves which lie outside the union, do not intersect each other except at $w_{0}$ and do not pass through any projection of branch points of the Riemannian image of $\Delta$ by $f(z)$. The elements of the inverse function $f^{-1}$ corresponding to $z^{\prime}$ and $z^{\prime \prime}$ can be continued analytically along these curves to their end points and further from them along radii of $C(0 ; \delta)$ and $C(\infty ; \delta)$, so that the curves in $\Delta$ corresponding to these continuations join each of $z^{\prime}$ and $z^{\prime \prime}$ to $\Gamma_{1}$ and $\Gamma_{3}$ and bound with parts of $\Gamma_{1}$ and $\Gamma_{3}$ a domain not containing $\Gamma_{2}$. This domain must be a subdomain of $\Delta$ and $f(z)$ must take the value 1 there; this is a contradiction. We see thus that $f(z)$ takes each value outside the union once and only once in $\Lambda$. 
Next we suppose that both of the cases (1) and (2) do not occur, that is, one of three points, say the point at infinity, is not contained in any one of $C_{1}, C_{2}$ and $C_{3}$ and one of these three discs is disjoint from the union of the other two. Then there is $z_{0}$ in $\Delta$ such that $f^{\prime}\left(z_{0}\right)$ lies outside the union of $C_{1}, C_{2}$ and $C_{3}$. The point $f\left(z_{0}\right)$ can be joined to the point at infinity with a curve $\Lambda$ lying outside this union, and we are led to a contradiction that the element of the inverse function $f^{-1}$ corresponding to $z_{0}$ can be continued analytically along $\Lambda$ up to a point arbitrarily near the point at infinity, so that $f(z)$ has a pole in $\Delta$. Thus we see that the denial of the case (1) implies the case (2). The proof is now complete.

5. Let $S_{n}$ denote the ring domain $\left|a_{n}\right|<|z|<\left|a_{n+1}\right|$, let $\Gamma_{n}$ denote the circle $|z|=\sqrt{\left|a_{n} a_{n+1}\right|}$ and let $\Delta_{n}$ denote the triply connected domain with boundary $\Gamma_{n} \cup\left\{a_{n+1}\right\} \cup \Gamma_{n+1}$. The harmonic modulus of $S_{n}$ is equal to $\log \left|a_{n+1}\right| a_{n} \mid$, so that we see from Lemma 1 that the diameter of the image of $\Gamma_{n}$ is dominated by $\delta_{n}=A \sqrt{\left|a_{n}\right| a_{n+1} \mid}$ and hence there is a spherical disc $C_{n}$ containing this image with radius less than $\delta_{n}$.

Lemma 3. Let $f(z)$ be meromorphic in the finite z-plane and omit three values 0,1 and $\infty$ outside $E$ and let $\delta>0$ be so small that the spherical discs $C(0 ; 2 \delta), C(1 ; 2 \delta)$ and $C(\infty ; 2 \delta)$ are mutually disjoint. If the points of $E$ satisfy the condition (*) and if there is only a finite number of $\Delta_{n}$ 's such that its three boundary components are mapped by $f(z)$ into $C(0 ; \delta)$, $C(1 ; \delta)$ and $C(\infty ; \delta)$ one by one, then $f(z)$ is rational.

Proof. From the condition (*), we obtain

$$
\left|\frac{a_{n+1}}{a_{n+2}}\right|=\mathrm{o}\left(\left|\frac{a_{n}}{a_{n+1}}\right|^{2}\right),
$$

so that $\delta_{n}$ tend to zero in the way that

$$
\delta_{n+1}=\mathrm{o}\left(\delta_{n}^{2}\right) \text {. }
$$

Therefore there is an $n_{1}$ such that

$$
\delta_{n}<\delta / 2 \text { for any } n \geqq n_{1} \text { and } \sum_{n=n_{1}}^{\infty} \delta_{n}<1 / 8 \text {. }
$$

Suppose now that there exists only a finite number of $\Delta_{n}$ such that their three boundary components are mapped into the discs $C(0 ; \delta), C(1 ; \delta)$ and $C(\infty ; \delta)$ one by one and denote by $n_{2}$ the maximum of $n$ taken over all such $\Delta_{n}$. Then we see by Lemma 2 that for any $n \geqq n_{0}=\max \left\{n_{1}, n_{2}\right\}$, the image of $\Delta_{n}$, consequently that of $\Delta_{n} \cup\left\{a_{n+1}\right\}$, is contained in a spherical disc $D_{n}$ with radius less than $2 \delta_{n}$. 
Since the union of $D_{n}\left(n \geqq n_{0}\right)$ is a connected set, its diameter with respect to the chordal distance is dominated by $2 \sum_{n=n_{0}}^{\infty} 2 \delta_{n} \leqq 4 \sum_{n=n_{0}}^{\infty} \delta_{n}<1 / 2$. By means of a linear transformation we can consider from this fact that $f(z)$ is bounded near the point at infinity. Hence $f(z)$ must be meromorphic in the extended $z$-plane, $|z| \leqq+\infty$, so that it is rational.

6. We now estimate the harmonic modulus of any ring domain contained in $\Delta_{n} \cup \Gamma_{n+1} \cup \Delta_{n+1}$ such that one connected component of its complement contains the point $a_{n+1}$ and the circle $\Gamma_{n}$ and the other contains the point $a_{n+2}$ and the circle $\Gamma_{n+2}$. Denoting by $\log \Psi(P / \varrho)(P, \varrho>0)$ the harmonic modulus of the normal domain of Teichmüller, the complement of the union of the two segments, $-\varrho \leqq x \leqq 0, y=0$ and $P \leqq x \leqq+\infty$, $y=0$ in the $z$-plane $(z=x+i y)$, we see that the harmonic modulus of our domain is dominated by $\log \Psi\left(\left|a_{n+2}\right| a_{n+1} \mid\right)$. It is well-known that

$$
\Psi(P / \varrho)<16 \frac{P}{\varrho}+8
$$

and we obtain the following

Lemma 4. The harmonic modulus of any ring domain considered above is dominated by $\log \left(32\left|a_{n+2}\right| a_{n+1} \mid\right)$.

7. Proof of the theorem. It is obviously sufficient if we can prove that the assumption of the existence of a function $f(z)$, single-valued, meromorphic and non-rational for $z \neq \infty$, and different from 0,1 and $\infty$ outside $E$, leads to a contradiction. Here we may assume without any loss of generality that $\left\{a_{n}\right\}$ consists only of zeros, 1-points and poles of $f(z)$, for otherwise, we delete from $\left\{a_{n}\right\}$ all other points and the remaining points also satisfy the condition (*).

We take $\delta>0$ so small that the discs $C(0 ; 2 \delta), C(1 ; 2 \delta)$ and $C(\infty ; 2 \delta)$ are mutually disjoint. Let $n_{0}$ be so large that $\delta_{n}=A \sqrt{\left|a_{n} / a_{n+1}\right|}$ $<\delta / 2$ for any $n \geqq n_{0}$. By Lemma 3 there is a $\Delta_{n}\left(n \geqq n_{0}\right)$ whose three boundary components are mapped into $C(0 ; \delta), C(1 ; \delta)$ and $C(\infty ; \delta)$ one by one, where we may assume that $f\left(\Gamma_{n+1}\right) \subset C(\infty ; \delta)$. Consider the quadruply connected domain $\Delta_{n} \cup \Gamma_{n+1} \cup \Delta_{n+1}$. The image of its boundary component $\Gamma_{n+2}$ is contained in some spherical disc $C_{n+2}$ with radius less than $\delta_{n+2}<\delta / 2$ and we see that $f(z)$ has a pole at $a_{n+2}$ and $C_{n+2}$ is contained in $C(\infty ; 2 \delta)$. In fact $f\left(a_{n+1}\right)=\infty$ or $C_{n+2}$ must contain the point at infinity and hence is contained in $C(\infty ; \delta)$, for otherwise, $f(z)$ must take the value $\infty$ in $\Delta_{n} \cup \Gamma_{n+1} \cup \Delta_{n+1}$. Suppose that $f\left(a_{n+1}\right)$ 
or $C_{n+2}$ is not contained in $C(\infty ; 2 \delta)$. Then there is a point $z \in \Delta_{n+1}$ whose image $f(z)$ lies in $C(\infty ; 2 \delta)-C(\infty ; \delta) \cup C_{n+2} \cup\left\{f\left(a_{n+1}\right)\right\}$ and can be joined the origin or the point $w=1$ with a path not intersecting the image of the boundary of $\Delta_{n+1}$, so that $f(z)$ takes the value 0 or 1 in $1_{n+1}$. Contradiction. Now it is obvious that $f(z)$ has a pole at $a_{n+2}$. Thus we can find a positive $d$ such that $d<2 \delta$ and the disc $C(\infty ; d)$ contains $C_{n+2}$.

Next we shall prove that $f(z)$ takes each value outside the union of the three discs $C(0 ; \delta), C(1 ; \delta)$ and $C(\infty ; d)$ once and only once in $\Delta_{n} U$ $\Gamma_{n+1} \cup \Delta_{n+1}$. By Lemma 2, $f(z)$ takes each value outside the union of $C(0 ; \delta), C(1 ; \delta)$ and $C(\infty ; \delta)$ once and only once in $\Delta_{n}$ so that the inverse image $\Gamma$ of the circle $[w, \infty]=2 \delta$ on $\Delta_{n}$ is a simple closed curve and separates $\Gamma_{n}$ and $a_{n+1}$ from $a_{n+2}$ and $\Gamma_{n+2}$. Now suppose that $f(z)$ takes a value $w_{0}$ outside the union of $C(0 ; \delta), C(1 ; \delta)$ and $C(\infty ; d)$ at two points $z^{\prime}$ and $z^{\prime \prime}$ in $\Delta_{n} \cup \Gamma_{n+1} \cup \Delta_{n+1}$, and join $w_{0}$ to $C(0 ; \delta)$ with a curve $\Lambda$ which lies outside the union and does not pass through any projection of branch points of the Riemannian image of $\Delta_{n} \cup \Gamma_{n+1}$ $\cup \Delta_{n+1}$ by $f(z)$. The elements of the inverse function $f^{-1}$ corresponding to $z^{\prime}$ and $z^{\prime \prime}$ can be continued analytically along $\Lambda$ to its end point and hence every value on $\Lambda$ is taken by $f(z)$ at least two times in $\Delta_{n} \cup \Gamma_{n+1}$ $\cup \Lambda_{n+1}$. Therefore we can assume that $w_{0}$ lies outside $C(\infty ; 2 \delta)$. Then one of $z^{\prime}$ and $z^{\prime \prime}$ must lie in the domain bounded by $\Gamma$ and $\Gamma_{n+2}$ and the corresponding element of $f^{-1}$ can be continued analytically to the origin along a curve outside $C(\infty ; 2 \delta)$, so that $f(z)$ takes the value 0 there; this contradicts our assumption.

Now we estimate $d$ from below. To this purpose we consider the annulus $R: 2<|w|<\sqrt{1-d^{2}} \mid d$ corresponding to the anıulus $1 / \sqrt{5}>[w, \infty]$ $>d$ on the Riemann sphere $\Sigma$, which separates $\mathrm{C}(0 ; \delta)$ and $C(1 ; \delta)$ from $C(\infty ; d)$. As we have seen above, the Riemannian image of $\Delta_{n} \mathrm{U}$ $\Gamma_{n+1} \cup \Delta_{n+1}$ covers $R$ univalently, the ring domain on $\Delta_{n} \cup \Gamma_{n+1} \cup \Delta_{n+1}$ corresponding to $R$ has the same harmonic modulus as $R$ and separates the boundary components $\Gamma_{n}$ and $a_{n+1}$ from the boundary components $a_{n+2}$ and $\Delta_{n+2}$. By Lemma 4 we have

$$
\text { har. mod. of } R=\log \left(\sqrt{1-d^{2}} / 2 d\right) \leqq \log \left(32\left|a_{n+2}\right| a_{n+1} \mid\right) \text {. }
$$

Since $d<2 \delta<\pi / 6$, we have the estimate that

$$
d \geqq\left(\sqrt{1-(\pi / 6)^{2}} / 64\right)\left|a_{n+1}\right| a_{n+2} \mid=\delta_{n+1}^{2} / \mathrm{M} \text {. }
$$

This implies that $C_{n+2}$ must intersect the disc $[w, \infty] \geqq \delta_{n+1}^{2} / M=\mathrm{m}$. Consider the domain $\Delta_{n+2}$. As mentioned in the proof of Lemma 3 ,

$$
\delta_{n+1}=o\left(\delta_{n}^{2}\right) \text {, }
$$


and we can suppose that $n$ is so large that

Then

$$
4 M \delta_{n+p+1} \leqq \delta_{n+p}^{2} \text { for each } p \geqq 1 .
$$

$$
\delta_{n+2} \leqq \delta_{n+1}^{2} / 4 M=m / 4
$$

and hence $C_{n+2}$, the disc containing the image of the boundary component $\Gamma_{n+2}$ of $\Delta_{n+2}$, cannot contain the point at infinity. Since the image of the boundary component $\Gamma_{n+3}$ of $\Delta_{n+2}$ is contained in a disc $C_{n+3}$ with radius less than $\delta_{n+3}<\delta_{n+2}$, we see by Lemma 2 that $C_{n+2}$ cannot be disjoint from $C_{n+3}$ and the image of $\Delta_{n+2} \cup\left\{a_{n+3}\right\}$ is contained in a spherical disc $D_{n+2} \supset C_{n+2} \cup C_{n+3}$ with radius less than $\delta_{n+2}+\delta_{n+3}<m / 2$. The disc $D_{n+2}$ cannot contain any one of the three points $w=0,1$ and $\infty$ on the one hand and the point $a_{n+3}$ must be a zero or a 1-point or a pole of $f(z)$ on the other; this is a contradiction and our theorem is established.

8. We shall be concerned here with the perfect case. Let $E$ be a Cantor set on the closed interval $I_{0}:-1 / 2 \leqq x \leqq 1 / 2, y=0$ on the real axis of the $z(=x+i y)$-plane with successive ratios $\xi_{n}, 0<\xi_{n}=2 l_{n}<2 / 3$. Defining the Cantor set $E$, we repeat successively to exclude an open segment from the middle of another segment and there remain $2^{n}$ segments of equal length $\Pi_{p=1}^{n} l_{p}$ after we repeat $n$ times, beginning with the interval $I_{0}$. We denote these segments by $I_{n, k}(n=1,2, \ldots ; k=1,2, \ldots$, $2^{n}$ ) and denoce by $S_{n, k}\left(n=1,2, \ldots ; k=1,2, \ldots, 2^{n}\right)$ the following annuli on the complementary domain $\Omega$ of $E$ :

$$
S_{n, k}=\left\{z ;\left(\Pi_{p=1}^{n} l_{p}\right)\left(1-l_{n+1}\right)<\left|z-z_{n, k}\right|<\left(\Pi_{p=1}^{n-1} l_{p}\right)\left(1-l_{n}\right) / 2\right\},
$$

where $z_{n, k}$ is the middle point of $I_{n, k}$. The harmonic modulus $\mu_{n}$ of $S_{n, k}$ is greater than $\log \left(2 / 3 \xi_{n}\right)$. We map $S_{n, k}$ conformally onto the annulus $1<|\eta|<e^{u_{n}}$ and consider the inverse image $\Gamma_{n, k}$ of the circle $|\eta|=e^{u_{n} / 2}$ on $S_{n, k}$. Supposing that $S_{n, k}$ encloses $S_{n+1,2 k-1}$ and $S_{n+1,2 k}$, we denote by $\Lambda_{n, k}$ the triply connected domain bounded by three curves $l_{n, k}^{\prime}$, $\Gamma_{n+1,2 k-1}$ and $\Gamma_{n+1,2 k}$.

We now prove

Theorem 2. If the successive ratios $\xi_{n}=2 l_{n}$ of a Cantor set $E$ satisfy the condition

$(* *) \quad \xi_{n+1}=0\left(\exp \left(-2^{n} / \Pi_{p=1}^{n} \xi_{p}\right)\right)$, i.e. $l_{n+1}=0\left(\exp \left(-1 / \Pi_{p=1}^{n} l_{p}\right)\right)$,

then $E$ is a Picard set in Lehto's sense.

9. As a consequence of Bohr-Landau's theorem we have

Lemma 5. Let $f(z)$ be single-valued regular and different from 0 and 1 outside $E$ and let $d_{n, k}$ be defined by 


$$
d_{n, k}=\min _{z \in \Gamma_{n, k}}[f(z), \infty]
$$

Then for sufficiently large $n$

$$
d_{n, k} \geqq d_{n}=\exp \left(-L / \sqrt{l_{n}}\left(\Pi_{p=1}^{n-1} l_{p}\right)\right),
$$

where $L>0$ depends only on $f(z)$.

Proof. Set

$$
M=\max _{\substack{z=x+i y \\-1 / 2 \leqq x \leqq 1 / 2, y=1}}|f(z)|
$$

and recall that $\Gamma_{n, k}$ is the circle $\left|z-z_{n, k}\right|=\left(\Pi_{p=1}^{n-1} l_{p}\right) \sqrt{l_{n}\left(1-l_{n}\right)\left(1-l_{n+1}\right) / 2}$. Then by Bohr-Landau's theorem, we have

$$
\left|f\left(z_{0}\right)\right| \leqq \exp \left(\frac{K \log (M+2)}{\left(\Pi_{p=1}^{n-1} l_{p}\right) \sqrt{l_{n}\left(1-l_{n}\right)\left(1-l_{n+1}\right) / 2}}\right),
$$

where $z_{0} \in \Gamma_{n, k}$ is the point satisfying $\mathfrak{R} z_{0}=\mathfrak{R} z_{n, k}$ and $\Im z_{0}>0$. $S_{n, k}$ is conformally equivalent to the annulus $1<|\eta|<e^{\mu_{n}}$ and hence the composite function $g(\zeta)=f\left(z\left(e^{\zeta}\right)\right)$ is regular and different from 0 and 1 and further has the period $2 \pi i$ in the strip domain $0<\Re \zeta<\mu_{n},-\infty<$ $\Im \zeta<+\infty$. Let $\zeta_{0}$ be a point whose image is $z_{0}$. Then any value taken by $f(z)$ on $\Gamma_{n, k}$ is taken by $g(\zeta)$ on the vertical segment $\Lambda$ of length $2 \pi$ with middle point $\zeta_{0}$, so that applying Bohr-Landau's theorem again, we have

$$
\sqrt{1-d_{n, k}^{2}} / d_{n, k}=\max _{\zeta \in \Lambda}|g(\zeta)| \leqq \exp \left(\frac{\left.K \log \left(\left|f\left(z_{0}\right)\right|+2\right)\right)}{1-2 \pi / \mu_{n}}\right) .
$$

Since $l_{n} \rightarrow 0$ and $\mu_{n} \rightarrow \infty$ as $n \rightarrow \infty$ from our assumption, we see that there is an $L>0$ depending only on $M$, consequently only on $f(z)$, such that

$$
a_{n, k} \geqq \exp \left(-L / \sqrt{l_{n}}\left(\Pi_{p=1}^{n-1} l_{p} ;\right)\right.
$$

for sufficiently large $n$.

10. For each $n$ and $k\left(n=1,2, \ldots ; k=1,2, \ldots, 2^{n}\right) \Delta_{n, k}$ is bounded by $\Gamma_{n, k}, \Gamma_{n+1,2 k-1}$ and $\Gamma_{n+1,2 k}$. Now we estimate the harmonic modulus of any ring domain in $\Delta_{n, k}$ one connected component of whose complement contains the boundary curve $\Gamma_{n, k}$ and the other contains $\Gamma_{n+1,2 k-1}$ and $\Gamma_{n+1,2 k}$. It is dominated by the harmonic modulus of the ring domain obtained when we delete the segment $I_{n, k}$ from the interior of $\Gamma_{n, k}$ and this domain is conformally equivalent to Grötzsch's normal domain $G_{P}, P=\left(2\left(1-l_{n}\right)\left(1-l_{n+1}\right)+l_{n}\right) /\left(2 \sqrt{\left.2 l_{n}\left(1-l_{n}\right)\left(1-l_{n+1}\right)\right)}\right.$ : the complementary domain of the union of the closed unit disc and the segment $P \leqq x \leqq+\infty, y=0$. Since 
har. mod. of $G_{P}=\log \Phi(P)$ and $\Phi(P) \leqq 4 P$

and since $l_{n} \rightarrow 0$ as $n \rightarrow \infty$, we have

Lemma 6. The harmonic modulus of any ring domain considered above is dominated by $\log \left(4 / \sqrt{l_{n}}\right)$ for sufficiently large $n$.

11. Proof of Theorem 2. Contrary to our assertion, let us suppose that there exists a single-valued meromorphic function $f(z)$ which has at least one essential singularity in $E$ and omits three values 0,1 and $\infty$ in the intersection of $\Omega$ and a neighbourhood of some singularity $\zeta_{0} \in E$. Since our argument given in the below is applicable locally, it will not bring any loss of generality if we give a contradiction under the stronger assumption that $f(z)$ omits the values 0,1 and $\infty$ in $\Omega$.

We delete from $E$ every point at which $f(z)$ is regular and takes a value different from 0 and 1 and denote by $E^{\prime}$ the resulting point set. Of course $\zeta_{0}$ belongs to $E^{\prime}$ and is not is olated because of Picard's theorem. Therefore there exist infinitely many $\Lambda_{n, k}$ such that the interior of one of its boundary curves $\Gamma_{n+1,2 k-1}$ and $\Gamma_{n+1,2 k}$ contains $\zeta_{0}$ and that of the other contains at least one point of $E^{\prime}$.

Let $\delta>0$ be so small that the discs $C(0 ; 2 \delta), C(1 ; 2 \delta)$ and $C(\infty ; 2 \delta)$ are mutually disjoint. By the condition $(* *)$ we can take $n_{0}$ so large that $\delta_{n}=A \sqrt{(3 / 2) \xi_{n}}<\delta / 12$ and $\xi_{n+1}<\xi_{n} / 4$ i.e. $\delta_{n+1}<\delta_{n} / 2$ for any $n \geqq n_{0}$, where $A$ is the constant of Lemma 1 . Since $\mu_{n}>\log \left(2 / 3 \xi_{n}\right)$, we see by Lemma 1 that the image of the circle $\Gamma_{n, k}, n \geqq n_{0}$, is contained in a spherical disc $C_{n, k}$ with radius less than $\delta_{n}<\delta / 12$. First we shall show that if the interior of $\Gamma_{n, k}, n \geqq n_{0}$, contains a point of $E^{\prime}$, then for this $\Gamma_{n, k}$, $C_{n, k}$ is contained in one of the three discs $C\left(0 ; 12 \delta_{n}\right), C\left(1 ; 12 \delta_{n}\right)$ and $C\left(\infty ; 12 \delta_{n}\right)$, which are mutually disjoint because $12 \delta_{n}<\delta$. In fact, suppose that our assertion is false and consider the domain $\Delta_{n, k}$. Then by Lemma 2 any one of $C_{n, k}, C_{n+1,2 k-1}$ and $C_{n+1,2 k}$ cannot be disjoint from the union of the other two, so that the image of $\Delta_{n, k}$ is contained in a spherical disc with radius less than $3 \delta_{n}$. Since this disc contains points outside the union of $C\left(0 ; 12 \delta_{n}\right), C\left(1 ; 12 \delta_{n}\right)$ and $C\left(\infty ; 12 \delta_{n}\right)$, it must lie completely outside the union of the dises $C\left(0 ; 6 \delta_{n}\right), C\left(1 ; 6 \delta_{n}\right)$ and $C\left(\infty ; 6 \delta_{n}\right)$. We consider the domain $\Delta_{n+1,2 k-1}$ which with $\Delta_{n, k}$ has $\Gamma_{n+1,2 k-1}$ as the common boundary. Each image of its three boundary curves is contained in a spherical disc with radius less than $\delta_{n+1}<\delta_{n} / 2$ and hence the same argument shows that the image of $\Delta_{n+1,2 k-1}$ is contained in a spherical disc with radius less than $3 \delta_{n+1}<3 \delta_{n} / 2$ and lying outside the union of the discs $C\left(0 ; 3 \delta_{n}\right), C\left(1 ; 3 \delta_{n}\right)$ and $C\left(\infty ; 3 \delta_{n}\right)$. The same holds for $\Delta_{n+1,2 k}$. Since for each $p \geqq 1$ 


$$
\delta_{n+p}<\delta_{n} / 2^{p}
$$

we can conclude by induction that the image of each domain $\Delta_{n+p, q}$ lying the interior of the simple closed curve $\Gamma_{n, k}$ is contained in a spherical disc with radius less than $3 \delta_{n} / 2^{p}$ and lying outside the union of the discs $C\left(0 ; 6 \delta_{n} / 2^{p}\right), C\left(1 ; 6 \delta_{n} / 2^{p}\right)$ and $C\left(\infty ; 6 \delta_{n} / 2^{p}\right)$. It follows that, in the interior of $\Gamma_{n, k}, f(z)$ takes values only in a spherical disc with radius less than

$$
\sum_{p=0}^{\infty} 3 \delta_{n} / 2^{p}<6 \delta_{n}
$$

and not containing the points $w=0,1$ and $\infty$. The set $E$ is of linear measure zero and hence each point of $E$ is removable for any bounded regular function (see Kametani [3]), so that $f(z)$ must be regular in the interior of $\Gamma_{n, k}$. Hence the interior of $\Gamma_{n, k}$ cannot contain any point of $E^{\prime}$; this is absurd.

Now we take a $\Delta_{n, k}, n \geqq n_{0}$, such that the interior of the boundary curve $\Gamma_{n, k}$ of $A_{n, k}$ contains $\zeta_{0}$. Then the interior of one of $\Gamma_{n+1,2 k-1}$ and $\Gamma_{n+1,2 k}$ say $\Gamma_{n+1,2 k-1}$, also contains $\zeta_{0}$ and as we have just seen, $C_{n, k}$ is contained in one of $C\left(0 ; 12 \delta_{n}\right), C\left(1 ; 12 \delta_{n}\right)$ and $C\left(\infty ; 12 \delta_{n}\right)$ and $C_{n+1,2 k-1}$ is contained in one of $C\left(0 ; 12 \delta_{n+1}\right), C\left(1 ; 12 \delta_{n+1}\right)$ and $C\left(\infty ; 12 \delta_{n+1}\right)$. Hence the following two cases are possible: (1) the centers of the discs containing $C_{n, k}$ and $C_{n+1,2 k-1}$ are distinct, or (2) they are identical. Let us suppose that the first case occurs. Obviously it does not bring any loss of generality if we assume that $C_{n, k}$ and $C_{n+1,2 k-1}$ are contained in $C\left(0 ; 12 \delta_{n}\right)$ and $C\left(\infty ; 12 \delta_{n+1}\right)$ respectively. Then we see that the disc $C_{n+1,2 k}$, the spherical disc containing the image of the last boundary curve $\Gamma_{n+1,2 k}$ of $\Delta_{n, k}$, contairis the point $w=1$ and hence is contained in $C\left(1 ; 12 \delta_{n+1}\right)$, for otherwise, $f(z)$ takes the value 1 in $\Delta_{n, k}$. By Lemma 2, $f(z)$ takes each value outside the union of $C\left(0 ; 12 \delta_{n}\right), C\left(1 ; 12 \delta_{n+1}\right)$ and $C\left(\infty ; 12 \delta_{n+1}\right)$ once and only once in $\Delta_{n, k}$. We consider here the quadruply connected domain $\Delta=\Delta_{n, k} \cup \Gamma_{n+1,2 k-1} \cup \Delta_{n+1,2 k-1}$. The images of its boundary curves $\Gamma_{n+2,4 k-3}$ nad $\Gamma_{n+2,4 k-2}$ are contained in the spherical discs $C_{n+2,4 k-3}$ and $C_{n+2,4 k-2}$ with radius less than $\delta_{n+2}<\delta_{n+1} / 2$ respectively. Of course the interior of one of $\Gamma_{n+2,4 k-3}$ and $\Gamma_{n+2,4 k-2}$, say $\Gamma_{n+2,4 k-3}$, contains $\zeta_{0}$. We suppose that the interior of $\Gamma_{n+2,4 k-2}$ contains a point of $E^{\prime}$. Then each of $C_{n+2,4 k-3}$ and $C_{n+2,4 k-2}$ is contained in one of $C\left(0 ; 12 \delta_{n+2}\right)$, $C\left(1 ; 12 \delta_{n+2}\right)$ and $C\left(\infty ; 12 \delta_{n+2}\right)$ and one of them contains the point at infinity, consequently is contained in $C\left(\infty ; 12 \delta_{n+2}\right)$, for otherwise, $f(z)$ takes the value $\infty$ in $A$. Hence the images of two of three boundary curves of $\Delta_{n+1,2 k-1}$, a triply connected subdomain of $\Delta$, is contained in $C\left(\infty ; 12 \delta_{n+1}\right)$, so that the image of the last boundary curve is also contained in $C\left(\infty ; 12 \delta_{n+1}\right)$ because of Lemma 2 . Thus both of $C_{n+2,4 k-3}$ and 
$C_{n+2,4 k-2}$ are contained in $C\left(\infty ; 12 \delta_{n+2}\right)$. Hence we can prove by the same argument as in the proof of Theorem 1 that $f(z)$ takes each value outside the union of $C\left(0 ; 12 \delta_{n}\right), C\left(1 ; 12 \delta_{n+1}\right)$ and $C\left(\infty ; 12 \delta_{n+2}\right)$ once and only once in 4 . Therefore if $d_{n+1}>12 \delta_{n+2}$ (by the condition (**) this is satisfied for every sufficiently large $n)$, the annulus $R, d_{n+1}>[w, \infty]>12 \delta_{n+2}$, which separates $C\left(0 ; 12 \delta_{n}\right)$ and $C\left(1 ; 12 \delta_{n+1}\right)$ from $C\left(\infty ; 12 \delta_{n+2}\right)$, corresponds to a ring domain on $\Delta$ which has the same harmonic modulus as it and separates $\Gamma_{n+2,4 k-3}$ and $\Gamma_{n+2,4 k-2}$ from the remaining boundary $\Gamma_{n, k} \cup \Gamma_{n+1,2 k}$ of $\Delta$. We see from Lemma 5 that this ring domain is contained in the subdomain $\Delta_{n+1,2 k-1}$ of $\Delta$, so that it separates $\Gamma_{n+2,4 k-3}$ and $\Gamma_{n+2,4 k-2}$ from the last boundary curve $\Gamma_{n+1,2 k-1}$ of $\Delta_{n+1,2 k-1}$. Hence by Lemma 6 we have that the harmonic modulus of $R$ is dominated by $\log \left(4 / \sqrt{l_{n+1}}\right)$, that is,

$$
d_{n+1} \sqrt{1-\left(12 \delta_{n+2}\right)^{2}} / 12 \delta_{n+2} \sqrt{1-d_{n+1}^{2}} \leqq 4 / \sqrt{l_{n+1}},
$$

while

$$
d_{n+1}=\exp \left(-L / \sqrt{l_{n+1}}\left(\Pi_{p=1}^{n} l_{p}\right)\right) \text { and } \delta_{n+2}=A \sqrt{3 l_{n+2}},
$$

so that

$$
\exp \left(-L / \sqrt{l_{n+1}}\left(\Pi_{p=1}^{n} l_{p}\right)\right) / 12 \sqrt{3} A \sqrt{l_{n+2}} \leqq 4 / \sqrt{l_{n+1}} .
$$

Recall our condition (**). Then we have, for some $M>0$,

$$
\sqrt{l_{n+1}} \exp \left(1 / \sqrt{l_{n+1}}\right) \leqq M \text {. }
$$

But this is impossible for any $n$ larger than some $n_{0}^{\prime}$ and we see that $\Gamma_{n+1,2 k}$ cannot surround any point of $E^{\prime}$ if $n \geqq n_{0}{ }^{\prime}$. Now suppose that $n \geqq n_{0}{ }^{\prime}$ and consider the quadruply connected domain $\Delta^{\prime}=\Delta \cup \Gamma_{n+2,4 k-3}$ $\cup \Delta_{n+2,4 k-3} \cup \Gamma_{n+2,4 k-2} \cup\left(\Gamma_{n+2,4 k-2}\right)$, where $\left(\Gamma_{n+2,4 k-2}\right)$ denotes the interior of the Jordan curve $\Gamma_{n+2,4 k-2}$. Then $f(z)$ does not take the values 0,1 and $\infty$ in $\Delta^{\prime}$. One of the boundary curves $\Gamma_{n+3,8 k-7}$ and $\Gamma_{n+3,8 k-6}$ of $\Delta^{\prime}$ surrounds $\zeta_{0}$ and only considering the annulus $d_{n+2}>[w, \infty]>12 \delta_{n+3}$ instead of $R$ in the above we have

$$
\sqrt{l_{n+2}} \exp \left(1 / \sqrt{l_{n+2}}\right) \leqq M
$$

if the other surrounds a point of $E^{\prime}$. Thus it cannot surround any point of $E^{\prime}$. Continuing this argument inductively, we are led to the contradiction that $\zeta_{0}$ is an isolated point of $E^{\prime}$, and follows that the case (1) is impossible for any $n \geqq n_{0}^{\prime}$.

Let us suppose that $n \geqq n_{0}{ }^{\prime}$. Then the case (2) must occur and we may assume here that $C_{n, k}$ and $C_{n+1,2 k-1}$ are contained in $\mathrm{C}\left(\infty ; 12 \delta_{n}\right)$ and $C\left(\infty ; 12 \delta_{n+1}\right)$ respectively. If the interior of the boundary curve $\Gamma_{n+1,2 k}$ 
of $\Delta_{n, k}$ contains a point of $E^{\prime}$, then $C_{n+1,2 k}$ is contained also in $C\left(\infty ; 12 \delta_{n+1}\right)$ because of Lemma 2. Therefore we see recalling Lemma 5 that each point of the annulus $R_{n}, d_{n}>[w, \infty]>12 \delta_{n+1}$, is covered the same times, say $v(n)$-times, by the Riemannian image of $\Delta_{n, k}^{\prime}$, where $\Delta_{n, k}^{\prime}$ denotes the domain $\Delta_{n, k} \cup \Gamma_{n+1,2 k} \cup\left(\Gamma_{n+1,2 k}\right)$ if $\Gamma_{n+1,2 k}$ does not surround any point of $E^{\prime}$ (the case $(i)$ ) and $A_{n, k}$ if it does so (the case (ii)). Consider the inverse image $\varrho_{n}$ of $R_{n}$. Any of its boundary curves cannot bound a subdomain of $\Delta_{n, k}^{\prime}$, for $f(z)$ takes the values 0 and 1 or the value $\infty$ in the domain bounded by such a curve if exists. Hence in the case (i), $\varrho_{n}$ is a ring domain separating the two boundary curves of $\Delta_{n, k}^{\prime}$, while in the case (ii), either it is a ring (the case (ii, a)) or triply connected (the case (ii, $b$ )) domain separating the boundary curve $\Gamma_{n, k}$ from the remaining $\Gamma_{n+1,2 k-1}$ and $\Gamma_{n+1,2 k}$, or it consists of two ring domains one of which separates $\Gamma_{n+1,2 k-1}^{\prime}$, consequently $\zeta_{0}$, from the remaining $\Gamma_{n, k}$ and $\Gamma_{n+1,2 k}$ and the other separates $\Gamma_{n+1,2 k}$ from $\Gamma_{n, k}$ and $\Gamma_{n+1,2 k-1}$ (the case (ii, c)). In this case we denote by $\varrho_{n}^{\prime}$ the former ring domain and by $v^{\prime}(n)$ the number of times that its Riemannian image covers $R_{n}$. Of course $v^{\prime}(n)<v(n)$. In the case (ii, b) we take $d_{n}{ }^{\prime}, d_{n}>d_{n}{ }^{\prime}>12 \delta_{n+1}$, so near to $12 \delta_{n+1}$ that the case (ii, c) occurs for the annulus $d_{n}{ }^{\prime}>[w, \infty]>12 \delta_{n+1}$, and for it, we define $\varrho_{n}^{\prime}$ and $\nu^{\prime}(n)$ in the same way as above. In other cases we put $\varrho_{n}{ }^{\prime}=\varrho_{n}$ and $\nu^{\prime}(n)=v(n)$.

In the cases (i), (ii, b) and (ii, c), we consider the ring or triply connected subdomain of $\Delta_{n, k}^{\prime} \cup \Gamma_{n+1,2 k-1} \cup{\Delta^{\prime}}_{n+1,2 k-1}$ placed between $\epsilon_{n}^{\prime}$ and $\varrho_{n+1}$ and see by the argument principle that $v(n+1)=v^{\prime}(n)$. Now we shall deal with the case (ii, a). First we note that one of the boundary curves $\Gamma_{n+2,4 k-1}$ and $\Gamma_{n+2,4 k}$ of $\Delta_{n+1,2 k}$, say $\Gamma_{n+2,4 k-1}$, surrounds a point of $E^{\prime}$ and hence the spherical disc $C_{n+2,4 k-1}$ is contained in one of $C\left(0 ; 12 \delta_{n+2}\right)$, $C\left(1 ; 12 \delta_{n+2}\right)$ and $C\left(\infty ; 12 \delta_{n+2}\right)$. We say that the case (ii, a') or (ii, a") occurs according as it lies in $C\left(\infty ; 12 \delta_{n+2}\right)$ or one of the other two. In the case (ii, a'), the interior of $\Gamma_{n+2,4 k}$ does not contain any point of $E^{\prime}$ or it does so and $C_{n+2,4 k}$ lies also in $C\left(\infty ; 12 \delta_{n+2}\right)$. Hence the inverse image of the annulus $R_{n+1}$ has at least one connected component in $A_{n+1,2 k}$, and the argument principle asserts that $v(n)$ is equal to the total number of times that all the Riemannian images of the components in $\Delta_{n+1,2 k}$ and $\Delta_{n+1,2 k}$ cover $R_{n+1}$, so that $v(n+1)<v(n)$. On the contrary, we have $v(n+1)=v(n)+1$ in the case (ii, $\left.\mathrm{a}^{\prime \prime}\right)$. In fact we see from Lemma 2 that $C_{n+2,4 k-1}$ and $C_{n+2,4 k}$ are contained in $C\left(0 ; 12 \delta_{n+2}\right)$ and $C\left(1 ; 12 \delta_{n+2}\right)$ one by one and the Riemannian image of $\Delta_{n+1,2 k}$ covers univalently the outside of the union $C\left(0 ; 12 \delta_{n+2}\right) \cup C\left(1 ; 12 \delta_{n+2}\right) \cup C\left(\infty ; 12 \delta_{n+1}\right)$. Hence for $\delta$ taken at the first part of the proof, the circle $[w, \infty]=\delta$, which separates $C\left(\infty ; 12 \delta_{n+1}\right)$ from $C\left(0 ; 12 \delta_{n+2}\right)$ and $C\left(1 ; 12 \delta_{n+2}\right)$, has as the inverse image in $\Delta_{n+1,2 k}$ a simple closed analytic curve $\Gamma$ separating $\Gamma_{n+1,2 k}$ from 
the remaining $\Gamma_{n+2,4 k-1}$ and $\Gamma_{n+2,4 k}$. Consider the domain placed between $\varrho_{n}, \varrho_{n+1}$ and $\Gamma$. Then the argument principle gives our formula.

Suppose that the case (ii, a) cannot occur except for a finite number of $n \geqq n_{0}{ }^{\prime}$. Since $\zeta_{0}$ is not isolated in $E^{\prime}$, the case (ii, b) or (ii, c) occurs for infinitely many $n$ 's, while the value of $v$ decreases strictly at such $n$ and hence must become negative; this is absurd. Thus the case (ii, a) occurs for infinitely many $n$ 's. Now let $n=n_{0}{ }^{\prime}+q$ be such one. By the Hurwitz formula, the Riemannian image of $\varrho_{n}$ has no branch point, so that $\varrho_{n}$ is conformally equivalent to the anrulus $d_{n}^{1 / v(n)} / \sqrt{d_{n}^{2 / \nu(n)}+\left(1-d_{n}^{2}\right)^{1 / v(n)}}$ $>[\omega, \infty]>\left(12 \delta_{n+1}\right)^{1 / v(n)} / \sqrt{\left(12 \delta_{n+1}\right)^{2 / v(n)}+\left(1-\left(12 \delta_{n+1}\right)^{2}\right)^{1 / v(n)}}$. Consequently by Lemma 6 we have

$$
d_{n} \sqrt{1-\left(12 \delta_{n+1}\right)^{2}} / 12 \delta_{n+1} \sqrt{1-d_{n}^{2}} \leqq\left(4 / \sqrt{l_{n}}\right)^{p(n)},
$$

that is,

$$
\exp \left(-L / \sqrt{l_{n}}\left(\Pi_{p=1}^{n-1} l_{p}\right)\right) / 12 \sqrt{3} A \sqrt{l_{n+1}} \leqq\left(4 / \sqrt{l_{n}}\right)^{p(n)} .
$$

Recall the condition $(* *)$. Since $v(n) \leqq v\left(n_{0}{ }^{\prime}\right)+q$ and $\left(4 / \sqrt{\bar{l}_{n}}\right)^{\nu(n)} \leqq$ $\left(4 / \sqrt{l_{n}}\right)^{v\left(n_{0}^{\prime}\right)+q}$, we have

$$
K^{1 /\left(v\left(n_{0}{ }^{\prime}\right)+q\right)} \exp \left(\left(1 / 2 \sqrt{l_{n}}-L\right) / \sqrt{l_{n}}\left(\Pi_{p=1}^{n-1} l_{p}\right)\left(v\left(n_{0}{ }^{\prime}\right)+q\right)\right)<4 / \sqrt{l_{n}},
$$

where $K$ is a positive constant. $K^{\left.1 /\left(n_{0}{ }^{\prime}\right)+q\right)} \rightarrow 1$ and $\left(\Pi_{p=1}^{n-1} l_{p}\right)\left(v\left(n_{0}{ }^{\prime}\right)+q\right) \rightarrow 0$ as $q \rightarrow \infty$, because $l_{p}<1 / 2$. Hence the quantity $\sqrt{l_{n}} \exp \left(1 / \sqrt{l_{n}}\right)$ must be dominated by a constant for infinitely many $n$; this is absurd and follows that the case (2) is also impossible for some $n \geqq n_{0}^{\prime}$.

Thus for some $A_{n, k}\left(n \geqq n_{0}^{\prime}\right)$ whose boundary curve $\Gamma_{n, k}$ surrounds $\zeta_{0}$, both of the cases (1) and (2) are impossible, while these two cases are all possible cases. This is caused by the assumpion of the existence of $f(z)$ different from 0,1 and $\infty$ in $\Omega$ and the theorem is established.

Mathematical Institute

Nagoya University

\section{References}

[1] Bohr, H. and Landau, E.: Über das Verhalten von $\zeta(s)$ und $\zeta_{k}(s)$ in der Nähe der Geraden $\sigma=1$ - Göttinger Nachr., (1910).

[2] Carleson, L.: A remark on Picard's theorem- Bull. Amer. Math. Soc., 67 (1961), $142-144$.

[3] Kametani, S.: On Hausdorff's measures and generalized capacities with some of their applications to the theory of functions- Jap. J. Math., 19 (1944-48), $217-257$. 
[4] LeHтo, O.: A generalization of Picard's theorem-Ark. Mat., 3nr. 45 (1958), 495-500.

[5] Matsumoto, K.: On exceptional values of meromorphic functions with the set of singularities of capacity zero-Nagoya Math. J., 18 (1961), 171-191.

[6] - - Some notes on exceptional values of meromorphic functions-Nagoya Math. J., 22 (1963), 189-201.

[7] —»- Existence of perfect Picard sets- Nagoya Math. J., 27 (1966), 213-222.

[8] -»- Perfect Picard set of positive capacity, to appear in Nagoya Math. J. 